\title{
Ueber die Eisensalze der extractiven Säuren aus Menschenharn.
}

\author{
Mit Schlussbemerkungen betreffend Hrn. E. Salkowsky's \\ „Beiträge zur Chemie des Harns." \\ Von
}

J. L. W. Thudichum,

in London.

Im Laufe meiner Untersuchungen über die Kryptophansäure (dieses Archiv, Seite 433) hatte ich gefunden, dass alle löslichen Salze derselben mit Eisenchlorid, in der Kälte oder bei Kochhitze, dicke braune Niederschläge geben, welche die organische Säure in Verbindung enthalten. Die löslichen Salze der Paraphansäure (einer Extractivsäure des Harns, die neben der Kryptophansäure vorkommt, und deren Untersuchung ich noch fortsetze) zeigen dieselbe Reaction. Ich hielt es daher für wahrscheinlich, dass die Eisenverbindungen dieser Säuren zu deren Isolirung aus dem Harn benutzt werden könnten, und dass die dann anderweitig gereinigten Säuren vermöge der grösseren Löslichkeit der Paraphanate von einander getrennt werden könnten. Diess waren die Absichten, welche mich zu den folgenden Versuchen führten. Dieselben haben meine Voraussetzungen vollkommen bestätigt, insofern grosse Mengen von kryptophan- und paraphansauren Salzen dabei erhalten wurden. Wie vorauszusehen war enthielten die Producte Hippurund Benzoesäure, die von den Extractivsäuren getrennt werden mussten. Aber ganz unerwartet war die Erscheinung, dass der Eisenniederschlag aus Harn beträchtliche Mengen von Substanzen enthielt, welche nach ihren weiteren Reactionen zur Gruppe der organischen Basen oder Alkaloide gehören, und sich durch mehrere diesen Körpern specifische Verbindungen von den extractiven Säuren beinahe vollständig trennen lassen. Die letzteren werde ich in einer zukünftigen Wittheilung über mehrere bisher unbekannte Alkaloide des Harns beschreiben. Im gegenwärtigen Artikel muss ich mich auf die Beschreibung der allgemeinen Verhältnisse 
der Eisenniederschläge, und ihrer Behandlung bis zur Darstellung von reiner Kryptophansäure beschränken.

Experiment 1. Eisensalz aus 16 Liter mit Kalk behandelten Harns; enthielt 26,39 Proc. Fe; gab ein Baryumsalz, welches durch fractionirte Fällung mit Alkohol Kryptophanat (und Paraphanat) lieferte.

16 Liter mit Kalkmilch behandelten Harns, concentrirt bis die Flüssigkeit 1,060 Sp. Gew. zeigte, wurden mit $430 \mathrm{Grm}$. Eisenchlorid in einem gleichen Gewicht Wasser gelöst, gemischt. Der Niederschlag wurde wohl ausgewaschen. Der nasse Schlamm wog $1760 \mathrm{Grm}$. 1,484 Grm. liessen bei $125^{\circ} 0,175 \mathrm{Grm}$. Rückstand, gleich 11,8 Proc. Die 0,175 Rückstand hinterliessen nach dem Glühen $0,066 \mathrm{Fe}_{2} \mathrm{O}_{3}$, gleich 26,39 Proc. Fe. Dem Schlamm wurden $80 \mathrm{Grm}$. Barythydrat in Wasser suspendirt zugesetat, die Mischung wurde erhitzt und filtrirt, und das Filtrat im Wasserbad verdampit. Das resultirende Baryumsalz wog $104 \mathrm{Grm}$. und enthielt, wie aus den späteren Analysen der Fractionen hervorgeht, ungefähr 29,3 Proc. Ba. Daraus folgt weiter, dass etwa $40 \mathrm{Grm}$. Baryt in einer unlöslichen Form in dem Eisenniederschlag zurückblieben, nachdem das gebildete Barytsalz soweit als möglich ausgewaschen worden war. Fractionirte Fällung des Baryumsalzes durch Alkohol.

$87 \mathrm{Grm}$. des Baryumsalzes wurden in etwa 700 Cc. Wasser gelöst, filtrirt und zu der Flüssigkeit wurden $220 \mathrm{Cc}$. Alkohol von 90 Procent gefügt. 'Der Niederschlag $[\mathrm{x}]$ wog im trocknen Zustand 4,0 Gramm.

0,0978 bei $110^{0}$ getrocknet gab $0,0557 \mathrm{BaSO}_{4}=33,48$ Proc. Ba. 0,2585 bei $125^{\circ}$ getrocknet gab $0,1495 \mathrm{BaSO}_{4}=34,00$ Proc. Ba. Der Niederschlag enthielt eine Spur Eisen.

Das Filtrat wurde mit 800 Cc. 90 procentigen Alkohols versetzt, und gab einen zweiten Niederschlag [y], welcher im trocknen Zastande $13,8 \mathrm{Grm}$. wog.

0,2230 bei $125^{\circ}$ gaben $0,1270 \mathrm{BaSO}_{4}=33,49$ Proc. Ba. Das Filtrat wurde mit zwei Liter Alkohol gemischt und gab einen dritten Niederschlag $\mathrm{Z}$, welcher bei $100^{\circ}$ getrocknet, $17 \mathrm{Grm}$. wog. 0,1169 bei $125^{\circ}$ gaben $0,0628 \mathrm{BaSO}_{4}=31,58$ Proc. Ba.

Das Filtrat wurde zur Trockne verdampft und der Rückstand mit [F] bezeichnet.

0,3140 bei $125^{0}$ gaben $0,1445 \mathrm{BaSO}_{4}=27,06$ Proc. Ba. 


\section{Uebersicht der Niederschläge.}

Fraction $1 \mathrm{x}$ wog 4,0 Grm. enthielt 33,7 Proc. Ba.

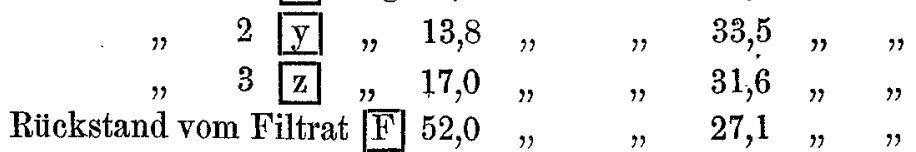
Aus diesen Daten kann man berechnen, dass die verwandten $87 \mathrm{Grm}$. Baryumsalz 29,2 Proc. Ba enthielten.

Weitere Fractionirang der Niederschläge $\mathrm{x}$ und $\mathrm{y}$.

Diese Niederschläge wurden vereinigt, in 80 Cc. Wasser gelöst, und nach Filtration von ein wenig unlöslicher Materie mit Alkohol behandelt; Niederschlag und Lösung wurden durch das Filter getrennt. Das Filtrat hinterliess beim Abdampfen nur einen geringen Rückstand. Der Niederschlag $(x+y)^{\prime}$ wurde bei $125^{\circ}$ getrocknet und analysirt.

$$
\begin{aligned}
& \text { 0,0758 gaben } 0,0448 \mathrm{BaSO}_{4}=34,75 \text { Proc. Ba. } \\
& 0,1525 \text { gaben } 0,0900 \mathrm{BaSO}_{4}=34,70 \text { Proc. Ba. }
\end{aligned}
$$

$(\mathrm{x}+\mathrm{y})^{\prime}$ wurde abermals in Wasser aufgelöst und durch Alkohol gefällt. Der Niederschlag $(x+y)^{\prime \prime}$ wurde bei $125^{\circ}$ getrocknet.

0,2205 gaben $0,1355 \mathrm{BaSO}_{4}=36,13$ Proc. Ba.

$(x+y)^{\prime \prime}$ wurde wieder in Wasser gelöst, filtrirt und das Filtrat mit Alkohol gefällt; der Niederschlag $(x+y)^{\prime \prime \prime}$ wurde bei $125^{\circ}$ getrocknet:

$$
\begin{aligned}
& \text { 0,0703 gaben } 0,0443 \mathrm{BaSO}_{4}=37,05 \text { Proc. Ba. } \\
& \text { 0,2720 gaben 0,1475 } \mathrm{BaCO}_{3}=37,70 \text { Proc. Ba. }
\end{aligned}
$$

Es erhellte aus diesen Versuchen, dass die zwei ersten Fractionen, den in Alkohol am wenigsten löslichen Theil des Salzes enthaltend, einen Körper enthielten, der mit jeder Lösung und Fällung seinen basischen Antheil vergrösserte, oder was dasselbe ist, mit jeder Lösung in Wasser und Fällung durch Alkohol organische Materie verlor.

Weitere Fractionirung des Niederschlags $\mathrm{Z}$.

Der Niederschlag $\mathrm{Z}$ wurde in $100 \mathrm{Cc}$. Wasser aufgelöst, die Lösung von einer geringen Truibung abfiltrirt, und mit $290 \mathrm{Ce}$. Alkohol von 90 Proc. gemischt. Der Niederschlag $z^{\prime}$ wurde bei $125^{\circ}$ getrocknet.

0,1505 gaben $0,0815 \mathrm{BaSO}_{4}=31,75$ Proc. Ba.

0,2075 gaben $\left(130^{\circ}\right) 0,1105 \mathrm{BaSO}_{4}=31,30$ Proc. Ba. 
z', 12 Grm. wiegend, wurde in 75 Ce. Wasser aufgelöst, und zu dieser Lösung wurden 75 Ce. Alkohol von 90 Proc. gemischt mit 75 Cc. Wasser gefügt. Der entstehende ganz geringe Niederschlag wurde abfiltrirt. Das klare Filtrat (dem eine gewisse Menge Alkohol von 90 Proc. zugesetzt werden konnte, ohne dass ein Niederschlag entstand) wurde durch Zusatz von viel Alkohol von 90 Proc. gefällt. Der Niederschlag $\mathrm{z}^{\prime \prime}$ wurde getrocknet.

0,2210 (bei $125^{\circ}$ ) gaben $0,1200 \mathrm{BaSO}_{4}=31,93$ Proc. Ba.

$\mathrm{z}^{\prime \prime}, 11,5 \mathrm{Grm}$. wiegend, wurde in wenig Wasser aufgelöst und durch einen Ueberschuss von 90 Proc. Alkohol gefällt. Der Niederschlag $\mathrm{z}^{\prime \prime \prime}$ wog $7,5 \mathrm{Grm}$.

0,2160 gaben $0,1170 \mathrm{BaSO}_{4}=30,13$ Proc. Ba.

0,3960 gaben $0,2025 \mathrm{BaSO}_{4}=30,07$ Proc. Ba. Demnach

war das Salz das zweibasische Kryptophanat, $\mathrm{Ba}^{\prime \prime}\left(\mathrm{C}_{10} \mathrm{H}_{16} \mathrm{~N}_{2} \mathrm{O}_{10}\right)$ welches 29,71 Proc. Ba erfordert.

Ueberführung von $z^{\prime \prime \prime}$ in Magnesiumsalz.

Die 7,5 Grm. $\mathrm{z}^{\prime \prime \prime}$ wurden in Wasser aufgelöst, die Lösung von einer Spur unlöslicher Materie abfiltrirt, hatte eine alkalische Reaction, und wurde mit einer Lösung von $5 \mathrm{Grm}$. Magnesium-Sulphat in ein wenig Wasser gefällt. Das Filtrat wurde dann vorsichtig mit einer verdïnnten Lösung von schwefelsaurem Magnesium versetzt, bis weder dieses Reagenz noch Barytwasser in dem Filtrat einen weiteren Niederschlag hervorbrachten. Ein Theil der Lösung wurde nun zur Trockne verdampft und bei $125^{\circ}$ ausgedörrt.

0,041 gaben nach dem Glühen $0,0061 \mathrm{MgO}=9,78$ Proc. Mg.

Daraus ging hervor, dass das Salz mehr Magnesium enthielt, als man nach dem verdrängten Baryum hätte erwarten sollen. Denn das Salz $\mathrm{Mg}^{\prime \prime}\left(\mathrm{C}_{10} \mathrm{H}_{16} \mathrm{~N}_{2} \mathrm{O}_{10}\right)$ fordert 6,8 Proc. $\mathrm{Mg}$; aber das dreibasische $\mathrm{Mg}_{3}\left(\mathrm{C}_{10} \mathrm{H}_{15} \mathrm{~N}_{2} \mathrm{O}_{10}\right)_{2}$ fordert 9,75 Proe. $\mathrm{Mg}$, was mit der gefundenen Menge übereinstimmt. Dies entspricht der aus der vorigen Abhandlung bekannten Tendenz der Magnesiumsalze alle ihre Basicitäten zu sättigen. Um diese Tendenz weiter zu fördern, wurde das Salz mit einem geringen Ueberschuss kaustischer Magnesia gekocht, filtrirt, verdampit und bei $125^{\circ}$ getrocknet.

0,1140 gaben $0,0220 \mathrm{Mg}=11,58$ Proc. $\mathrm{Mg}$.

0,3960 gaben $0,0735 \mathrm{Mg}=12,14$ Proc. $\mathrm{Mg}$.

Das Salz war demnach vierbasisches Kryptophanat mit zwei Molekeln Hydratwasser, $\mathrm{C}_{10} \mathrm{H}_{14} \mathrm{Mg}_{2} \mathrm{~N}_{2} \mathrm{O}_{10}+2 \mathrm{H}_{2} \mathrm{O}$, welches 11,83 Proc. $\mathrm{Mg}$ 
verlangt, die Lösung des Salzes gab die folgenden Reactionen: mit Eisenchlorid reichlichen Niederschlag;

mit Chromchlorid keinen Niedersehlag;

mit Kupferacetat einen Niederschlag beim Kochen,

löslich im Ueberschus des Acetats, durch Alkohol gefällt;

mit Bleiacetat einen Niederschlag, leicht löslich im Ueberschuss, aber durch Alkohol fällbar.

Goldlösung wurde beim Kochen reducirt, aber nicht Platinchlorid.

Uebersicht der Fractionen des Baryumsalzes (aus Eisensalz), welche durch Alkohol gefällt wurden.

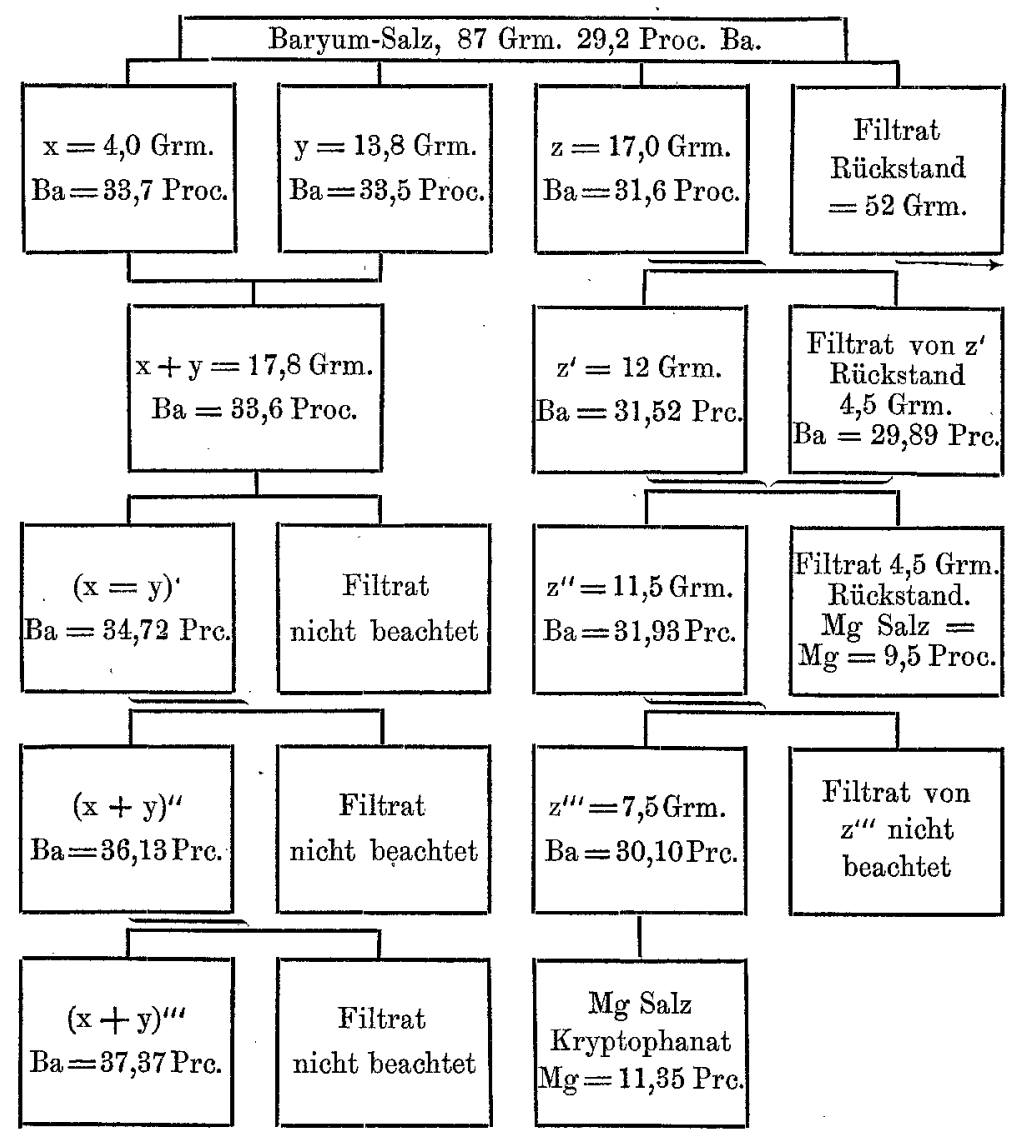

Das Filtrat von z' liess einen Rückstand beim Abdampfen, welcher trocken $4,5 \mathrm{Grm}$. wog.

0,1966 bei $110^{\circ}$ gaben $0,1009 \mathrm{BaSO}_{4}=30,18$ Proc. Ba. 
0,4165 bei $130^{\circ}$ gaben $0,2097 \mathrm{BaSO}_{1}=29,60$ Proc. Ba. Die übrige Substanz wurde in $40 \mathrm{Cc}$. Wasser gelöst, zu dem $60 \mathrm{Cc}$. 90 procentigen Alkohols gesetzt wurden. Es bildete sich kein Niederschlag. Es wurden dann weitere $100 \mathrm{Cc}$. Alkohol zugesetzt. Ein reichlicher Niederschlag entstand, welcher zu $\mathbf{z}^{\prime}$ gefügt wurde. Der trockne Rïckstand von dem verdampften Filtrat enthielt 30,4 Procent Ba.

Das Filtrat von Z" liess einen Niederschlag, welcher trocken 4,5 Grm. wog. Er wurde mit Schwefelsäure genau zersetzt, und das Filtrat mit Ueberschuss von Magnesia gekocht und filtrirt. Das Filtrat wurde verdampit, und das Magnesiumsalz bei $120^{\circ}$ getrocknet.

$$
\begin{aligned}
& 0,1980 \text { gaben } 0,0317 \mathrm{MgO}=9,6 \text { Proc. } \mathrm{Mg} \text {. } \\
& 0,3586 \text { gaben } 0,0568 \mathrm{MgO}=9,48 \text { Proc. } \mathrm{Mg} \text {. }
\end{aligned}
$$

Das Salz wurde abermals mit Magnesia gekocht und bei $125^{\circ}$ getrocknet.

$$
0,2285 \text { gaben } 0,0365 \mathrm{MgO}=9,6 \text { Proc. } \mathrm{Mg} \text {. }
$$

Das Product schien eine Mischung von kryptophansaurer mit paraphansaurer Magnesia zu sein.

Experiment 2. Der Harn wurde filtrirt, verdampit, mit Kalk behandelt, und abermals filtrirt. In diesem Zustande hatte die Flïssigkeit Sp. G. 1,060, und mass 10 Liter. Sie wurde mit einer Lösung von Eisenchlorid in seinem eignen Gewicht Wasser gefällt. Der Eisenniederschlag ẁurde mit 17 Liter Wasser gewaschen. Der Schlamm wog $372 \mathrm{Grm}$. 1,352 Grm. desselben bei $125^{\circ}$ getrocknet, hinterliessen 0,686 Rückstand $=51,5$ Proc. Die 0,686 Grm. liessen nach Glühhitze 0,213 Grm. $\mathrm{Fe}_{2} \mathrm{O}_{3}=21,73$ Proc. Fe. Der Schlamm wurde jetzt mit heisser Barytlösung behandelt. Die 161,6 Grm. des (trocken gedachten) Eisensalzes gaben nur $37 \mathrm{Grm}$. Baryumsalz. Folglich blieb ein beträchtlicher Theil der organischen extractiven Säure bei dem Eisen, und konnte mit Baryt nicht ausgezogen werden. Dieser Theil kann, wie aus anderen Experimenten erhellt, nur dureh Behandlung des Eisenniederschlages mit Schwefelammonium erhalten werden.

Experiment 3. 11 Liter Harn auf ein Drittel konzentrirt, gaben ein Eisensalz mit 28,12 Proe. Fe. Aus diesem wurde durch Kochen mit Baryt ein erstes Baryumsalz erhalten. Die Lösung dieses Salzes in etwa $180 \mathrm{Cc}$. Wasser wurde mit soviel Alkohol versetzt, dass das Volum der Mischung 500 Cc. betrug; der ent- 
stehende Niederschlag wurde abfiltrirt, und das Filtrat verdampit. Sobald es nur 50 Cc. betrug, wurde es mit $50 \mathrm{Cc}$. Alkohol von 90 Proc. gemischt. Es entstand kein Niederschlag. Darauf wurde Alkohol von 95 Proc. zugesetzt bis die Mischung 300 Cc. mass. Es entstand ein zusammenschmelzender Niederschlag, von dem die Flüssigkeit abgegossen wurde.

A. Der Niederschlag wurde in $50 \mathrm{Cc}$. Wasser aufgelösst, und die Lösung mit 40 Cc. Alkohol von 95 Proc. gemischt. Ein geringer entstehender Niederschlag wurde entfernt. Das Filtrat wurde mit viel Alkohol von 95 Proc. 'gemischt, und der entstandene Niederschlag bei $125^{\circ}$ getrocknet.

0,1952 gaben 0,0972 $\mathrm{BaSO}_{4}=29,43$ Proc. Ba.

B. Das Filtrat wurde auf dem Dampfbad eingeengt, und ein Theil bei $125^{\circ}$ getrocknet.

$$
0,1680 \text { gaben } 0,0725 \mathrm{BaSO}_{4}=25,37 \text { Proc. Ba. }
$$

Die Menge von $B$ schien viel mehr zu betragen als die von $A$.

Was von $B$ iubrig war wurde bei $100^{\circ}$ getrocknet, und mit wenig Alkohol von 95 Proc. behandelt. Es löste sich etwas auf $(\beta)$ und ein Theil blieb ungelöst $(\alpha)$.

a) Der unlösliche Theil wurde bei $125^{\circ}$ getrocknet. 0,1610 gaben $0,0830 \mathrm{BaSO}_{4}=30,31$ Proc. Ba.

Das ganze wurde in $10 \mathrm{Cc}$. Wasser gelöst, und $20 \mathrm{Cc}$. Alkohol von 95 Proc. zugefügt; es entstand kein Niederschlag. Weitere 90 Ce. Alkohol gaben sogleich Niederschlag, der sich aber bei Zusatz von wenig Wasser beinahe ganz löste. Was unlöslich blieb wurde weggeworfen; das Filtrat wurde verdampit und bei $125^{\circ}$ getrocknet.

0,1320 gaben $0,0710 \mathrm{BaSO}_{4}=31,63$ Proc. Ba.

B) Der löslich'e Theil, welcher mit Alkohol von 95 Proc. ansgezogen worden war, wurde verdampit, und abermals in Alkohol von derselben Stärke aufgelöst. Ein geringer unlöslicher Rückstand wurde entfernt. Das Filtrat wurde verdampit, und der Rückstand bei $125^{\circ}$ getrocknet.

0,0734 gaben $0,0225 \mathrm{BaSO}_{4}=18,02$ Proc. Ba.

$\mathrm{Z}$ weites Baryumsalz. Diess wurde durch eine zweite $\mathrm{Ab}$ kochung des Eisenniederschlags mit frischem Barytwasser erhalten. Der konzentrirte Auszug: wurde mit verdünntem Alkohol behandelt, um die in schwachem Weingeist unlöslichen Salze zu entfernen, und dann zu Syrupdicke abgedampft. Dieser wurde mit Alkohol 
von 90 Proc. behandelt. Man erhielt einen Niederschlag und ein Filtrat.

Das Filtrat wurde verdampit, und der Riickstand abermals mit Alkohol von 90 Proc. behandelt; die von einem geringen Niederschlag getrennte Lösung wurde wieder auf ein kleines Volum gebracht und nun mit Alkohol von 90 Proc. behandelt, bis dieser keinen Niederschlag mehr hervorbrachte. Das auf diese Weise erhaltene Baryumsalz (welches in viel Alkohol von 95 Proc. löslich war) wurde bei $125^{\circ}$ getrocknet.

0,1600 gaben 0,0316 $\mathrm{BaSO}_{4}=11,61$ Proc. Ba,

Experiment 4. Eisensalz wird in Baryumsalz, und dieses theilweise in Magnesium, Silber und Quecksilbersalz verwandelt.

Etwa 25 Liter filtrirten Harns wurden auf ein Drittel konzentrirt, kalt mit Kalk behandelt, filtrirt und mit Eisenchlorid im Ueberschuss behandelt, bis Ammoniak einen unmittelbaren Niederschlag in dem Filtrat hervorbrachte. Der abfiltrirte Niederschlag: wurde mit neun Liter Wasser gewaschen. Die teigige Masse wog 900 Grm: und enthielt 9,25 Proc. Rückstand bei 100 getrocknet, also im Ganzen 83,25 Grm. troeknes Eisensalz. Die Masse wurde auf dem Dampfbad mit 55 Grm. Barytkrystallen erhitzt, worauf sie zu einem kleinern Volum einschrumpite. Es wurden nun mehr Baryt, und 400 Ce. Wasser zugesetzt, und nach dem Mischen wurde filtrirt.

Das Filtrat 900 Cc. war alkalisch. Der rückständige Eisenniedersehlag wurde mit mehr Wasser gekocht und filtrirt; das Filtrat, etwa $200 \mathrm{Cc}$. messend war ebenfalls alkalisch. Die vereinten Filtrate $(1100 \mathrm{Cc}$.) wurden zu Syrupdicke eingedampft. Der Syrup mass 78 Ce. und wog 101 Grm., daher sein Sp. Gew. $=1,295$ Grm. Man liess ihn zwanzig Stunden stehen und dekantirte ihn dann von einem geringen Absatz. 0,3225 gaben 0,159 festen Rückstand bei $110^{\circ}$, desshalb ganzer fester Riuckstand ungefähr 50 Grm. Spätere Auszuige desselben Eisensalzes gaben noch 14 Grm. Baryumsalz. Die ganze Menge trocknes Baryumsalz, welche aus 83,25 Grm. trocknen Eisensalzes erhalten wurde, war demnach ungefähr 64 Grm. (aus 25 Liter Harn, also 2,56 Grm. aus jedem Liter). Das Baryumsalz wurde bei $125^{0}$ getrocknet und analysirt, um die darin enthaltenen Elemente kennen zu lernen. 
1) 0,4815 gaben $0,2335 \quad \mathrm{BaSO}_{4}=28,51$ Proc. Ba.

2) $0,3790 " 0,1842 \quad " \quad=28,51 \quad "$,

3) $0,4745 " 0,2315 "=28,71 " n$

4) $0,4830 " 0,6150 \quad \mathrm{CO}_{2}=34,84$ Proc. C.

5) $0,3225,0,4025, \quad=34,04 \quad, "$,

6) $0,4830 " 0,1750 \quad \mathrm{H}_{2} \mathrm{O}=4,26$ Proc. $\mathrm{H}$.

7) $0,3225 ", \quad 0,1125, \quad=3,88 "$,

8) $0,2353 " 0,01792 \mathrm{~N}=7,62$ Proc. N.

9) $0,4815,0,03692, \quad=7,67, "$,

Im lufttrocknen gepulverten Zustand enthielt das Salz 21,3 Proc. $\mathrm{Ba}$, denn

$$
0,1875 \text { gaben } 0,0680 \mathrm{BaSO}_{4},=21,3 \text { Proc. Ba. }
$$

1,6 Grm. dieses Salzes wurden mit 25 Cc. Alkohol von 90 Proe. behandelt, und das alkoholische Filtrat wurde verdampft und bei $100^{\circ}$ getrocknet. Es gab 0,0075 $\mathrm{BaSO}_{4}$. Daraus ging hervor, dass Alkohol von der angegebenen Stärke nur sehr wenig aus dem Baryumsalz auszog.

Mit Bleizucker verhielt sich das Baryumsalz wie das Kryptophanat.

Magnesiumsalz. Das in Alkohol unlösliche Salz wurde in Wasser gelöst, und durch verdiunnte Schwefelsäure von Baryt befreit; die freie Säure wurde durch Kochen mit Ueberschuss von Magnesia mit dieser Base verbunden, und das Salz bei $125^{\circ}$ getrocknet.

$$
\text { 0,2400 gaben 0,0490 } \mathrm{MgO}=12,25 \text { Proc. } \mathrm{Mg} \text {. }
$$

Silbersalz. Durch doppelte Zersetzung mit Silbernitrat gab dieses Magnesiumsalz ein gut aussehendes Silbersalz.

Quecksilbersalz. 18 Grm. des Baryumsalzes wurde in Wasser aufgelöst, und die Lösung wurde mit starkem Alkohol gemischt. Der Niederschlag wurde in Wasser aufgelöst, und die Lösung gekocht um den Alkohol ganz auszutreiben. Nach dem Filtriren wurde Merkurinitrat $\left(\mathrm{Hg}^{\prime \prime}\left(\mathrm{NO}_{3}\right)_{2}\right)$ in Lösung zu dem alkalischen Salz gefügt. Es entstand ein weisser Niederschlag. Es wurde jetzt etwas kohlensaures Natron za der Mischung gesetzt, bis dieselbe eine schwach alkalische Reaction hatte, und dann etwas sehr verdünnte Salpetersäure; Wasser mit Salpetersäure angesäuert wurde zum Waschen des Niederschlags verwandt. Derselbe bei $125^{\circ}$ getrocknet enthielt in 100 Theilen $47,90 \mathrm{Hg}$.

1) Die Analysen 4 und 6 , sowie 5 und 7 gehören zusammen als gleichzeitige Resultate von zwei Verbrennungen. 
Experiment 5. Eisensalz wird in Kalisalz verwandelt. Löslichkeit des Eisensalzes in Aetzkali.

640 Grm. nassen Eisensalzteiges, 85 Grm. trocknen Salzes enthaltend, wurden mit 40 Grm. Aetzkali behandelt, die Mischung verwandelte sich in eine dunkel gefärbte Lösung, die sich anf dem Dampfbad anfangs in ein festes Gele verwandelte, dann aber in eine Flutssigkeit und Eisenoxyd spaltete. Nach dem Filtriren von Eisenoxyd war die Lösung frei von Eisen. Sie wurde verdampft bis sie 109 Grm. wog, und enthielt dann 33,37 Grm. trocknen Kalisalzes.

2,041 gaben 0,606 Rückstand bei $120^{\circ}=29,7$ Proc. Rückstand. 0,606 gaben $0,344 \mathrm{~K}_{2} \mathrm{CO}_{3}=30,63$ Proc. $\mathrm{K}$.

Das ganze Product wurde mit wenig Wasser und 400 Ce. Alkohol von 90 Proc. gemischt. Fs bildeten sich zwei Lagen, eine untere wässrige, von etwa $50 \mathrm{Cc}$. Volum, und eine obere spirituöse, die letztere wurde verdampft and hinterliess $25 \mathrm{Grm}$. trocknen Riückstandes.

Die untere wässrige Schicht wurde mit drei Volumen Wasser verdünt, filtrirt und verdampit. Ein Theil wurde mit Schwefelsäure behandelt, ohne unlösliche freie Säure zu liefern. Das übrige wurde mit Merkurinitrat in Quecksilbersalz verwandelt. Die Flüssigkeit wurde dabei sauer erhalten, sodass kaustisches Kali im Uebersehuss darin nur eine Trübung, und keinen Niederschlag von Oxyd hervorbrachte.

Der erste Quecksilberniederschlag war gefärbt, und wurde nicht beachtet, sondern entfernt. Mit mehr Merkurinitrat wurde jetzt ein weisser Niederschlag erhalten.

Experiment 6. Fünf Eisenniederschläge werden nacheinander aus derselben Flüssigkeit dargestellt; nur die zwei ersten enthalten Extractivsäuren.

Eine gewisse Menge Harn wurde filtrirt, aufs halbe Volum verdampft, abgekühlt, mit Kalk gemischt und filtrirt. $\mathrm{Zu}$ dem Filtrat wurde Eisenchlorid gesetzt bis Ammoak sogleich in einen Niederschlag von Eisenoxydhydrat in dem Filtrat hervorbrachte; der Eisenniederschlag wurde abfiltrirt, und mit Wasser gewaschen (Erster Eisenniederschlag). 
Ueber die Eisensalze der extractiven Säuren aus Menschenharn. 465

Das Filtrat gab beim Kochen einen zweiten braunen Niederschlag (Zweiter Eisenniedersehlag).

Das Filtrat von diesem, welches beim Kochen keinen weiteren Niederschlag gab, wurde mit Ammoniak in geringem Ueberschuss behandelt, wobei es șich dunkel grün fürbte, und bei weiterem Zusatz von Ammoniak setzte es einen grünlichschwarzen Niederschlag ab. (Dritter Eisenniederschlag.) Durch weiteren Zusatz von Ammoniak und Kochen wurde ein vierter Eisenniederschlag von dunkelbrauner Farbe erhalten. Das Filtrat war jetzt dunkelbraun gefärbt, und gab auf Zusatz von Alkohol einen f ünften Eisenniederschlag, das Filtrat war jetzt nur blassgelb, und enthielt wenig feste Stoffe.

Der dritte Niederschlag enthielt nur wenig organische Materie und bestand hauptsächlich aus Oxydoxydul, mit etwas Oxydulphosphat. Der vierte Niederschlag war hauptsächlich Oxydhydrat. Der fünfte Niederschlag bestand hauptsächlich aus unorganischen Materien, basischem Eisenchlorid; Potassiumchlorid und Sulphat; und enthielt auch Omicholin and Omicholsäure mit Eisen verbunden, aber kein Sodium.

Die beiden ersten Niederschläge wurden in Baryumsalz verwandelt. Ein Theil desselben - wurde abermals mit Eisenchlorid in drei aufeinanderfolgenden Fractionen gefällt.

Fraction 1. 0,5451 liessen $0,0995 \mathrm{Fe}_{2} \mathrm{O}_{3}=12,76$ Proc. Fe.

Fraction 2. 0,2025 liessen $0,0385 \mathrm{Fe}_{2} \mathrm{O}_{3}=13,30$ Proc. Fe.

Fraction 3. 0,1958 liessen $0,0404 \mathrm{Fe}_{2} \mathrm{O}_{3}=14,44$ Proc. Fe.

Diese Resultate liessen keinen Zweifel, dass der organische Körper in dem Baryumsalz noch eine Mischung war. Das ganze Baryumsalz wurde daher behandelt wie folgt. Es wurde mit Salzsäure zersetzt (Schwefelsäure zeigte sich als unbranchbar) ein harziger Niederschlag fiel nieder, davon ein Theil in Alkohol löslich, ein anderer unlöslich war. Beide waren in Aether unlöslich. Ausserdem fiel ein weisser Niederschlag von Benzoesäure, welcher durch Aether ausgezogen wurde; der Aether enthielt auch ein wenig dem Omicholin ähnliche Substanz. Es blieb nun eine saure Lösung, welche mit Aetzkali und Barytwasser neutralisirt, filtrirt, und dann mit Eisenchlorid gefällt wurde.

Gereinigtes Eisensalz. 0,3250 liessen $0,0765 \mathrm{Fe}_{2} \mathrm{O}_{3}=$ 16,48 Proc. Eisen.

Dieses Salz wurde abermals durch Kochen mit kaustischem 
Baryt zersetzt; das Filtrat wurde kochend mit Kohlensäure behandelt, filtrirt, und zur Trockne verdampit. Das Salz' war beinahe farblos.

$$
\begin{aligned}
& 0,2140 \text { gaben } 0,1305 \mathrm{BaSO}_{4}=35,85 \text { Proc. Ba. } \\
& 0,4555 \text { gaben } 0,2755 \mathrm{BaSO}_{4}=35,54 \text { Proc. Ba. }
\end{aligned}
$$

Dieses Salz entspricht daher dem dreibasischen Kryptophanat, enthält aber wahrscheinlich noch ein wenig Paraphanat, davon es befreit werden muss. Fractionirte Fällungen mit Eisenchlorid und fractionirte Fällungen mit Alkohol werden vielleicht am besten zum Ziele führen.

Experiment 7. Eisensalz und Ammoniakprocess.

Das Eisensalz wurde aus konzentrirtem mit Kalk behandeltem Harn erhalten. Es wurde sorgfältig gewaschen und löste sich dann a uf dem Filter in kaustischem Ammoniak auf, sodass die dunkelrothe Lösung durchs Filter lief. Beim Kochen und Verdampfen fiel beinahe alles Eisen (eine Spur blieb gelöst) heraus. Die Lösung des Ammoniaksalzes wurde vermittelst des Bleiprocesses behandelt, und lieferte viel reines Kryptophanat.

Aus diesen Experimenten lassen sich folgende Schlüsse ziehen:

1) Der Harn des Menschen enthält beträchtliche Mengen von sogenannten Extractivstoffen, stickstoffhaltige Süuren, welche ich Kryptophan- und Paraphansäure genannt habe.

2) Dieselben können aus dem mit Kalk, oder besser mit Baryt alkalisch gemachten, auf ein Viertel konzentrirten Harn, durch Eisenchlorid vollständig gefällt werden. Die Mischung nimmt dabei eine starksanre Reaction an.

3) Das Eisensalz kann durch kaustischen Baryt, kaustisches Kali, oder Ammoniak zersetzt werden. Baryt zersetzt das Salz nicht vollständig, liefert aber das reinere Product. Schwefelammonium zersetzt das Eisensalz ebenfalls, allein die resultirende Kryptophansäure wird etwas schwefelhaltig.

4) Das Eisensalz enthält Hippur- oder Benzoesäure, meistens nur die letztere.

5) Das Eisensalz enthält ferner ein Alkaloid, das von der Kryptophansäure ete. durch einen directen Process getrennt werden kann, den ich in einer späteren Mittheilung beschreiben werde. 
Schlussbemerkungen betreffend Herrn E. Salkowsky's „Beiträge zur Chemie des Harns".

In Band 2, S. 354 dieses Archivs 1869 hat Herr E. Salkowsky eine „Untersuchung des Niederschlags den Eisenchlorid, im Harn nach Ausfällen der Phosphorsäure verursacht" veröffentlicht. Er erhielt ein wenig Harnsäure; zwei gefärbte Stoffe in geringer Menge; durch Destilliren des kohlensauren Natron-Auszugs des Eisenniederschlags von 22 Liter Harn mit Weinsäure etc. 0,101 Grm. Natronsalz einer flüchtigen Säure; dann aus 35 Liter Harn auf ähnliche Weise 0,2230 Grm. Barytsalz, von dem er annimmt, dass es Propionsäure enthalten habe. Allein von den Extractivstoffen, welche er zu finden ausging, hat er keine Spur entdeckt. Wenn man die grossen Mengen derselben bedenkt, welche er aus 57 Liter Harn hätte erhalten haben mïssen, und welche er, obwohl ihm unbewusst unter den Händen hatte, so sieht man wie es selbst dem Suchenden geschehen kann, vor lauter Bäumen den Wald nicht zu sehen. Auch der Fingerzeig, welchen sein Niederschlag b) hätte geben können, verliert er aus den Augen, und obwohl er die Identität seines branen Pulvers mit meinem Uromelanin erkennt (und die seines in Alkohol löslichen rothbraunen Pulvers mit meinem Uropittin hätte erkennen sollen), so ist ihm doch ,die chemische Individualität des Uromelanins nicht gentigend begründet". Er vermeidet daher die Zusammensetzung der Substanzen, oder ihrer Verbindungen mit Metalloxyden zu ermitteln, injizirt sie aber Kaninchen ins Unterhautzellgewebe. Diese letzteren Experimente nun haben Angesichts der Hauptfrage nicht den geringsten Werth, und sind in der That nur eine Ausflucht, um mit den erhaltenen Substanzen, die zum Reinigen, Analysiren und Verbinden mit Metalloxyden offenbar viel zu geringfügig waren, doch etwas anzustellen. Allein derartige Experimente sind viel leichter als Darstellungen und Reinigungen chemischer Individuen, leichter als Elementaranalysen, leichter als Darstellung and Analysen von Verbindungen mit Metalloxyden, und stellen am Ende doch einen physiologisehen Heroismus vor. Und dieser Heroismus befähigt dann auch wahrscheinlich Herrn E. Salkowsky, ohne Forschung, ohne Vergleiche, ohne Analysen gemacht zu haben, dem Uromelanin die chemische Individualität abzusprechen. Und dergleichen 
nennt man „Beiträge zur Chemie des Harns"! Und derselbe Kritiker, welcher dem nach allen Richtungen durch und durch analysirten Uromelanin die chemische Individualität abspricht, verlangt oder erwartet, dass man eine einzige und auf die Voraussetzung nicht einmal gut passende Barytbestimmung als Berechtigung für ihn betrachte, das Bischen Substanz, in welchem dieser Baryt enthalten war, als fast reinen propionsauren Baryt anzunehmen! Diess ist wohl "Sic vos non vobis" ins Wissenschaftliche übersetzt. Derartige Lucubrationen tragen ihren Titel nur aus Courtoisie, dem „lueus a non lucendo" ähnlich heissen sie „Beiträge" weil sie zur Vermehrung unsrer Kenntnisse wirklich nichts beitragen.

\section{Abwehr der Verdächtigungen, welche Herr Neubauer zu Wiesbaden betreffs der Kryptophansäure veröffentlicht hat.}

Von

\section{J. L. W. Thudiehum}

in London.

S. 53 seiner „Anleitung zur Analyse des Harns" gibt Herr Neubauer einen Paragraphen in kleinen Diamanttypen, die Krytophansäure behandelnd. Er gibt keine Formel, keine Angabe über die elementare Zusammensetzung, keinerlei Nachricht von den Verbindungen oder dem sonstigen Verhalten der Säure. Nachdem er im Anfang das wohlfeile Mittel des Fragezeichens in Klammer gebraucht hat, schliesst er seine Relation mit einem Versuch zu einem Sarkasmus, und dann mit den Worten - „allein weitere Untersuchungen müssen noch entscheiden, ob wir es hier in der That wirklich" (der Pleonasmus gehört Herrn Neubauer) "mit einer reinen Substanz zu thu haben, was nach der beschriebenen Darstellungsmethode nicht über jeden Zweifel erhaben ist." Allein 